\title{
On Rectifying Dual Space Curves
}

\author{
Ahmet YüCESAN, Nihat AYYILDIZ, \\ and A. Ceylan ÇÖKEN \\ Süleyman Demirel University \\ Department of Mathematics \\ 32260 Isparta - Turkey \\ yucesan@fef.sdu.edu.tr ayyildiz@fef.sdu.edu.tr \\ ceylan@fef.sdu.edu.tr
}

Received: October 23, 2006

Accepted: April 26, 2007

\begin{abstract}
We give some characterizations of the rectifying curves in the dual space and show that rectifying dual space curves can be stated with the aid of dual unit spherical curves. Thus, we have a link between rectifying dual space curves and classical surfaces in the Euclidean three-space.

Key words: dual space, rectifying dual space curve, Frenet formulae, dual Darboux vector.

2000 Mathematics Subject Classification: 53A04, 53A25, 53A40.
\end{abstract}

\section{Introduction}

In the Euclidean three-space $\mathbb{R}^{3}$, lines combined with one of their two directions can be represented by unit dual vectors over the ring of dual numbers. The most important properties of real vector analysis are valid for the dual vectors. The oriented lines in $\mathbb{R}^{3}$ are in one-to-one correspondence with the points of a dual unit sphere. A dual point on dual unit sphere in $\mathbb{D}^{3}$ corresponds to a line in $\mathbb{R}^{3}$ and two different points on $\mathbb{D}^{3}$ represent two skew-lines in $\mathbb{R}^{3}$ in general. A differentiable curve on dual unit sphere in $\mathbb{D}^{3}$ represents a ruled surface in $\mathbb{R}^{3}$ (see $[4,5]$ ).

A curve in $\mathbb{R}^{3}$ whose position vector always lies in its rectifying plane is called a rectifying curve. A useful method of determining rectifying curves in the Euclidean three-space $\mathbb{R}^{3}$ has been developed by Chen [1]. He shows that it can be possible to determine completely all rectifying curves in $\mathbb{R}^{3}$. 
Chen and Dillen [2] find a relationship between rectifying curves and the centrodes given by the endpoints of the Darboux vectors of a space curve and playing an important role in mechanics. The centrode of a space curve with constant curvature and nonconstant torsion is a rectifying curve and vice versa. Moreover, there exists an inequality for the geodesic curvature of the central projection of a space curve into a unit sphere where equality holds exactly for a rectifying curve.

In this paper, by using the method in [1], we have characterizations of rectifying curves in the dual space $\mathbb{D}^{3}$. In section 2 , we characterize rectifying dual space curves with respect to the distance function, the components of the position vector and harmonic curvature of dual space curve. In the last section, we show that rectifying dual space curves can be stated with the aid of dual unit spherical curves.

Therefore, this work gives a link with the classical surface theory since a differentiable curve on the dual unit sphere corresponds to a ruled surface in $\mathbb{R}^{3}$.

\section{Preliminaries}

By a dual number $\hat{x}$, we mean an ordered pair of the form $\left(x, x^{*}\right)$ for all $x, x^{*} \in \mathbb{R}$. Let the set $\mathbb{R} \times \mathbb{R}$ be denoted as $\mathbb{D}$. Two inner operations and an equality on $\mathbb{D}=\left\{\left(x, x^{*}\right) \mid x, x^{*} \in \mathbb{R}\right\}$ are defined as follows:

(i) $\oplus: \mathbb{D} \times \mathbb{D} \rightarrow \mathbb{D}$ for $\hat{x}=\left(x, x^{*}\right), \hat{y}=\left(y, y^{*}\right)$ defined as

$$
\hat{x} \oplus \hat{y}=\left(x, x^{*}\right) \oplus\left(y, y^{*}\right)=\left(x+y, x^{*}+y^{*}\right)
$$

is called the addition in $\mathbb{D}$.

(ii) $\odot: \mathbb{D} \times \mathbb{D} \rightarrow \mathbb{D}$ for $\hat{x}=\left(x, x^{*}\right), \hat{y}=\left(y, y^{*}\right)$ defined as

$$
\hat{x} \odot \hat{y}=\hat{x} \hat{y}=\left(x, x^{*}\right) \odot\left(y, y^{*}\right)=\left(x y, x y^{*}+x^{*} y\right)
$$

is called the multiplication in $\mathbb{D}$.

(iii) If $x=y, x^{*}=y^{*}$ for $\hat{x}=\left(x, x^{*}\right), \hat{y}=\left(y, y^{*}\right) \in \mathbb{D}, \hat{x}$ and $\hat{y}$ are equal, and it is indicated as $\hat{x}=\hat{y}$.

If the operations of addition, multiplication and equality on the set $\mathbb{D}=\mathbb{R} \times \mathbb{R}$ with set of real numbers $\mathbb{R}$ are defined as above, the set $\mathbb{D}$ is called the dual numbers system and the element $\left(x, x^{*}\right)$ of $\mathbb{D}$ is called a dual number. In a dual number $\hat{x}=\left(x, x^{*}\right) \in \mathbb{D}$, the real number $x$ is called the real part of $\hat{x}$ and the real number $x^{*}$ is called the dual part of $\hat{x}$. The dual number $(1,0)=1$ is called unit element of multiplication operation in $\mathbb{D}$ or real unit in $\mathbb{D}$. The dual number $(0,1)$ is to be denoted with $\varepsilon$ in short, and the $(0,1)=\varepsilon$ is to be called dual unit. In accordance with the definition of the operation of multiplication, it can easily be seen that $\varepsilon^{2}=0$. Also, the dual number $\hat{x}=\left(x, x^{*}\right) \in \mathbb{D}$ can be written as $\hat{x}=x+\varepsilon x^{*}($ see $[6,7])$.

The set of $\mathbb{D}=\left\{\hat{x}=x+\varepsilon x^{*} \mid x, x^{*} \in \mathbb{R}\right\}$ of dual numbers is a commutative ring according to the operations 
(i) $\left(x+\varepsilon x^{*}\right)+\left(y+\varepsilon y^{*}\right)=(x+y)+\varepsilon\left(x^{*}+y^{*}\right)$,

(ii) $\left(x+\varepsilon x^{*}\right)\left(y+\varepsilon y^{*}\right)=x y+\varepsilon\left(x y^{*}+y x^{*}\right)$.

The dual number $\hat{x}=x+\varepsilon x^{*}$ divided by the dual number $\hat{y}=y+\varepsilon y^{*}$ provided $y \neq 0$ can be defined as

$$
\frac{\hat{x}}{\hat{y}}=\frac{x+\varepsilon x^{*}}{y+\varepsilon y^{*}}=\frac{x}{y}+\varepsilon \frac{x^{*} y-x y^{*}}{y^{2}} .
$$

The set of

$$
\begin{aligned}
& \mathbb{D}^{3}=\mathbb{D} \times \mathbb{D} \times \mathbb{D}=\left\{\overrightarrow{\hat{x}} \mid \overrightarrow{\hat{x}}=\left(x_{1}+\varepsilon x_{1}^{*}, x_{2}+\varepsilon x_{2}^{*}, x_{3}+\varepsilon x_{3}^{*}\right)\right. \\
& =\left(x_{1}, x_{2}, x_{3}\right)+\varepsilon\left(x_{1}^{*}, x_{2}^{*}, x_{3}^{*}\right) \\
& \left.=\vec{x}+\varepsilon \overrightarrow{x^{*}}, \vec{x}, \overrightarrow{x^{*}} \in \mathbb{R}^{3}\right\}
\end{aligned}
$$

is a module on the ring $\mathbb{D}$. For any $\overrightarrow{\hat{x}}=\vec{x}+\varepsilon \overrightarrow{x^{*}}, \overrightarrow{\hat{y}}=\vec{y}+\varepsilon \overrightarrow{y^{*}} \in \mathbb{D}^{3}$, the scalar or inner product and the vector product of $\overrightarrow{\hat{x}}$ and $\overrightarrow{\hat{y}}$ are defined by, respectively,

$$
\begin{gathered}
\langle\overrightarrow{\hat{x}}, \overrightarrow{\hat{y}}\rangle=\langle\vec{x}, \vec{y}\rangle+\varepsilon\left(\left\langle\vec{x}, \overrightarrow{y^{*}}\right\rangle+\left\langle\overrightarrow{x^{*}}, \vec{y}\right\rangle\right), \\
\overrightarrow{\hat{x}} \wedge \overrightarrow{\hat{y}}=\left(\hat{x}_{2} \hat{y}_{3}-\hat{x}_{3} \hat{y}_{2}, \hat{x}_{3} \hat{y}_{1}-\hat{x}_{1} \hat{y}_{3}, \hat{x}_{1} \hat{y}_{2}-\hat{x}_{2} \hat{y}_{1}\right),
\end{gathered}
$$

where $\hat{x}_{i}=x_{i}+\varepsilon x_{i}^{*}, \hat{y}_{i}=y_{i}+\varepsilon y_{i}^{*} \in \mathbb{D}, 1 \leq i \leq 3$. If $x \neq 0$, the norm $\|\overrightarrow{\hat{x}}\|$ of $\overrightarrow{\hat{x}}=\vec{x}+\varepsilon \overrightarrow{x^{*}}$ is defined by

$$
\|\overrightarrow{\hat{x}}\|=\sqrt{\langle\overrightarrow{\hat{x}}, \overrightarrow{\hat{x}}\rangle}=\|\vec{x}\|+\varepsilon \frac{\left\langle\vec{x}, \overrightarrow{x^{*}}\right\rangle}{\|\vec{x}\|} .
$$

A dual vector $\overrightarrow{\hat{x}}$ with norm 1 is called a dual unit vector. Let $\overrightarrow{\hat{x}}=\vec{x}+\varepsilon \overrightarrow{x^{*}} \in \mathbb{D}^{3}$. The set

$$
\mathbb{S}^{2}=\left\{\overrightarrow{\hat{x}}=\vec{x}+\varepsilon \overrightarrow{x^{*}} \mid\|\overrightarrow{\hat{x}}\|=(1,0) ; \quad \vec{x}, \overrightarrow{x^{*}} \in \mathbb{R}^{3}\right\}
$$

is called the dual unit sphere with the center $\hat{O}$ in $\mathbb{D}^{3}$.

If every $x_{i}(t)$ and $x_{i}^{*}(t), 1 \leq i \leq 3$, real valued functions, are differentiable, the dual space curve

$$
\begin{aligned}
\hat{x}: I \subset \mathbb{R} \longrightarrow \mathbb{D}^{3} & \\
t \longrightarrow \overrightarrow{\hat{x}(t)} & =\left(x_{1}(t)+\varepsilon x_{1}^{*}(t), x_{2}(t)+\varepsilon x_{2}^{*}(t), x_{3}(t)+\varepsilon x_{3}^{*}(t)\right) \\
& =\overrightarrow{x(t)}+\varepsilon \overrightarrow{x^{*}(t)}
\end{aligned}
$$

in $\mathbb{D}^{3}$ is differentiable. We call the real part $\overrightarrow{x(t)}$ the indicatrix of $\overrightarrow{x(t)}$. The dual arc length of the curve $\overrightarrow{\hat{x}(t)}$ from $t_{1}$ to $t$ is defined as

$$
\hat{s}=\int_{t_{1}}^{t}\left\|(\overrightarrow{\hat{x}(t)})^{\prime}\right\| d t=\int_{t_{1}}^{t}\left\|(\overrightarrow{x(t)})^{\prime}\right\| d t+\varepsilon \int_{t_{1}}^{t}\left\langle\vec{t},\left(\overrightarrow{x^{*}(t)}\right)^{\prime}\right\rangle d t=s+\varepsilon s^{*},
$$


where $\vec{t}$ is a unit tangent vector of $\overrightarrow{x(t)}$. From now on we will take the arc length $s$ of $\overrightarrow{x(t)}$ as the parameter instead of $t$.

Now we will obtain equations relative to the derivatives of dual Frenet vectors throughout the curve in $\mathbb{D}^{3}$. Let

$$
\begin{aligned}
& \hat{x}: I \longrightarrow \mathbb{D}^{3} \\
& s \longrightarrow \overrightarrow{\hat{x}(s)}=\overrightarrow{x(s)}+\varepsilon \overrightarrow{x^{*}(s)}
\end{aligned}
$$

be a $C^{4}$ curve with the arc length parameter $s$ of the indicatrix. Then,

$$
\frac{d \overrightarrow{\hat{x}}}{d \hat{s}}=\frac{d \overrightarrow{\hat{x}}}{d s} \frac{d s}{d \hat{s}}=\overrightarrow{\hat{t}}
$$

is called the dual unit tangent vector of $\overrightarrow{\hat{x}(s)}$. With the aid of equation (1), we have

$$
\hat{s}=s+\varepsilon \int_{s_{1}}^{s}\left\langle\vec{t},\left(\overrightarrow{x^{*}}\right)^{\prime}\right\rangle d s
$$

and from this $\frac{d \hat{s}}{d s}=1+\varepsilon \Delta$, where the prime denotes differentiation with respect to the arc length $s$ of indicatrix and $\Delta=\left\langle\vec{t},\left(\overrightarrow{x^{*}}\right)^{\prime}\right\rangle$. Since $\overrightarrow{\hat{t}}$ has constant length 1 , its differentiation with respect to $\hat{s}$, which is given by

$$
\frac{d \overrightarrow{\hat{t}}}{d \hat{s}}=\frac{d \overrightarrow{\hat{t}}}{d s} \frac{d s}{d \hat{s}}=\frac{d^{2} \overrightarrow{\hat{x}}}{d \hat{s}^{2}}=\hat{\varkappa} \overrightarrow{\hat{n}},
$$

measures the way the curve is turning in $\mathbb{D}^{3}$. The norm of the vector $\frac{d \overrightarrow{\hat{t}}}{d \hat{s}}$ is called curvature function of $\overrightarrow{\hat{x}(s)}$. We impose the restriction that the function $\hat{\varkappa}: I \rightarrow \mathbb{D}$ is never pure dual. Then, the dual unit vector $\overrightarrow{\hat{n}}=\frac{1}{\hat{\varkappa}} \frac{d \overrightarrow{\hat{t}}}{d \hat{s}}$ is called the principal normal of $\overrightarrow{\hat{x}(s)}$. The dual vector $\overrightarrow{\hat{b}}$ is called the binormal of $\overrightarrow{\hat{x}(s)}$. We call the dual vectors $\overrightarrow{\hat{t}}, \overrightarrow{\hat{n}}, \overrightarrow{\hat{b}}$ the dual Frenet trihedron of $\overrightarrow{\hat{x}(s)}$ at the point $\hat{x}(s)$. The equalities relative to derivatives of dual Frenet vectors $\overrightarrow{\hat{t}}, \overrightarrow{\hat{n}}, \overrightarrow{\hat{b}}$ throughout the dual space curve are written in the matrix form

$$
\frac{d}{d \hat{s}}\left[\begin{array}{l}
\hat{t} \\
\overrightarrow{\hat{n}} \\
\overrightarrow{\hat{b}}
\end{array}\right]=\left[\begin{array}{ccc}
0 & \hat{\varkappa} & 0 \\
-\hat{\varkappa} & 0 & \hat{\tau} \\
0 & -\hat{\tau} & 0
\end{array}\right]\left[\begin{array}{l}
\overrightarrow{\hat{t}} \\
\overrightarrow{\hat{n}} \\
\overrightarrow{\hat{b}}
\end{array}\right],
$$

where $\hat{\varkappa}=\varkappa+\varepsilon \varkappa^{*}$ is nowhere pure dual curvature and $\hat{\tau}=\tau+\varepsilon \tau^{*}$ is nowhere pure dual torsion. The formulae (2) are called the Frenet formulae (see [6]). The planes spanned by $\{\overrightarrow{\hat{t}}, \overrightarrow{\hat{b}}\},\{\overrightarrow{\hat{t}}, \overrightarrow{\hat{n}}\}$, and $\{\overrightarrow{\hat{n}}, \overrightarrow{\hat{b}}\}$ at each point of the dual space curve are called the rectifying plane, the osculating plane, and the normal plane, respectively. 
Since the position vector of a rectifying dual space curve lies in the rectifying plane of the curve, the position vector can be written as

$$
\overrightarrow{\hat{x}(s)}=\hat{\lambda}(s) \overrightarrow{\hat{t}(s)}+\hat{\mu}(s) \overrightarrow{\hat{b}(s)}
$$

for some dual functions $\hat{\lambda}(s)$ and $\hat{\mu}(s)$.

\section{Some characterizations of rectifying curves in $\mathbb{D}^{3}$}

Now we characterize rectifying curves in dual space $\mathbb{D}^{3}$.

Theorem 2.1. Let $\hat{x}: I \rightarrow \mathbb{D}^{3}$ be a rectifying curve in dual space $\mathbb{D}^{3}$ with $\hat{\varkappa}=\varkappa+\varepsilon \varkappa^{*}$ such that $\varkappa>0$ and let $\hat{s}$ be dual arc length of the dual space curve $\hat{x}$. Then the following statements are valid:

(i) The dual distance function $\hat{\rho}=\|\overrightarrow{\hat{x}(s)}\|$ satisfies $\hat{\rho}^{2}=\hat{s}^{2}+\hat{c}_{1} \hat{s}+\hat{c}_{2}$ for some dual constants $\hat{c}_{1}$ and $\hat{c}_{2}$.

(ii) The tangential component of the position vector of the dual space curve is given by

$$
\langle\overrightarrow{\hat{x}(s)}, \overrightarrow{\hat{t}(s)}\rangle=\hat{s}+\hat{k},
$$

where $\hat{k}$ is a dual constant.

(iii) The normal component $\hat{x}^{N}$ of the position vector of the dual space curve is of dual constant length and the dual distance function $\hat{\rho}$ is nonconstant.

(iv) The torsion $\hat{\tau}=\tau+\varepsilon \tau^{*}$ is nowhere pure dual, that is $\tau \neq 0$, and the binormal component $\langle\overrightarrow{\hat{x}(s)}, \overrightarrow{\hat{b}(s)}\rangle$ of the position vector is a dual constant.

Conversely, if $\hat{x}: I \rightarrow \mathbb{D}^{3}$ is a curve in $\mathbb{D}^{3}$ with $\hat{\varkappa}=\varkappa+\varepsilon \varkappa^{*}$, where $\varkappa>0$, and if one of (i), (ii), (iii), or (iv) holds, then $\hat{x}$ is a rectifying dual space curve.

Proof. Let us first suppose that $\hat{x}: I \rightarrow \mathbb{D}^{3}$ is parameterized by the dual arc length function $\hat{s}$. Then, the position vector $\vec{x}(s)$ of the dual space curve $\hat{x}$ satisfies the equation

$$
\overrightarrow{\hat{x}(s)}=\hat{\lambda}(s) \overrightarrow{\hat{t}(s)}+\hat{\mu}(s) \overrightarrow{\hat{b}(s)}
$$

for some dual functions $\hat{\lambda}(s)$ and $\hat{\mu}(s)$. Differentiating equation (3) with respect to the dual arc length function $\hat{s}$ and using the Frenet equations (2), we get

$$
\frac{d}{d \hat{s}} \hat{\lambda}(s)=(1,0)=1+\varepsilon 0=1, \quad \hat{\lambda}(s) \hat{\varkappa}(s)=\hat{\tau}(s) \hat{\mu}(s), \quad \frac{d}{d \hat{s}} \hat{\mu}(s)=0 .
$$


Hence, from equations (4) we obtain that $\hat{\lambda}(s)=\hat{s}+\hat{k}$ for some dual constant $\hat{k}=k+\varepsilon k^{*}$, where $k, k^{*} \in \mathbb{R}$, and that $\hat{\mu}(s)=d u a l$ constant. On the other hand, since

$$
\hat{\lambda}(s) \hat{\varkappa}(s)=(s+k) \varkappa+\varepsilon\left(\left(s^{*}+k^{*}\right) \varkappa+(s+k) \varkappa^{*}\right) \neq 0,
$$

the dual constant $\hat{\mu}(s)$ is nonzero. Taking into consideration equation (3), we have

$$
\langle\overrightarrow{\hat{x}(s)}, \overrightarrow{\hat{t}(s)}\rangle=\hat{s}+\hat{k} .
$$

Thus we have proved statement (ii).

Now, if we take the derivative of

$$
\hat{\rho}^{2}=\langle\overrightarrow{\hat{x}(s)}, \overrightarrow{\hat{x}(s)}\rangle
$$

with respect to the dual arc length function $\hat{s}$ and use equation (5), we find

$$
\hat{\rho}^{2}=\hat{s}^{2}+\hat{c}_{1} \hat{s}+\hat{c}_{2},
$$

where $\hat{c}_{1}$ and $\hat{c}_{2}$ are dual constants. Hence, statement (i) is obtained.

From equation (3) it is clear that the normal component $\hat{x}^{N}$ of the position vector of the dual space curve is given by equation

$$
\langle\overrightarrow{\hat{x}(s)}, \overrightarrow{\hat{b}(s)}\rangle=\hat{\mu}(s) .
$$

Here, since $\hat{\mu}(s)$ is a dual constant, we get that the normal component $\hat{x}^{N}$ of the position vector of the dual space curve has a dual constant length. Thus, statement (iii) is proved.

And finally, from the dual constancy of $\hat{\mu}$, the fact that $\varkappa>0$, the relation $\hat{\lambda}(s)=\hat{s}+\hat{k}$, and the second equation of (4) we have statement (iv).

Conversely, assume that statement (i) or statement (ii) holds. Then, we have $\langle\overrightarrow{\hat{x}(s)}, \overrightarrow{\hat{t}(s)}\rangle=\hat{s}+\hat{k}, \hat{k} \in \mathbb{D}$. Differentiating this equation with respect to $\hat{s}$, we obtain $\hat{\varkappa}\langle\overrightarrow{\hat{x}(s)}, \overrightarrow{\hat{n}(s)}\rangle=0$. Since $\varkappa>0$ by assumption, $\langle\overrightarrow{\hat{x}(s)}, \overrightarrow{\hat{n}(s)}\rangle=0$ is found. Hence, the dual space curve $\hat{x}$ is rectifying.

Now, suppose that statement (iii) holds. Then, we have

$$
\langle\overrightarrow{\hat{x}(s)}, \overrightarrow{\hat{x}(s)}\rangle=\langle\overrightarrow{\hat{x}(s)}, \overrightarrow{\hat{t}(s)}\rangle^{2}+\hat{c},
$$

where $\hat{c}$ is a dual constant. Differentiation of this equation with respect to $\hat{s}$ shows that

$$
\langle\overrightarrow{\hat{x}(s)}, \overrightarrow{\hat{t}(s)}\rangle=(1+\hat{\varkappa}\langle\overrightarrow{\hat{x}(s)}, \overrightarrow{\hat{n}(s)}\rangle)\langle\overrightarrow{\hat{x}(s)}, \overrightarrow{\hat{t}(s)}\rangle .
$$

On the other hand, since the distance function $\hat{\rho}$ is a nonconstant, we get $\langle\overrightarrow{\hat{x}(s)}, \overrightarrow{\hat{t}(s)}\rangle \neq 0$. Moreover, from equation (6) and the assumption that $\varkappa>0$, we obtain $\langle\overrightarrow{\hat{x}(s)}, \overrightarrow{\hat{n}(s)}\rangle=0$, which means that $\hat{x}$ is a rectifying dual space curve.

Finally, if statement (iv) holds, then by using Frenet equations (2), we easily obtain that the dual space curve $\hat{x}$ is a rectifying dual space curve. 
The Frenet formulae given by (2) can be interpreted kinematically as follows: if a moving point moves along a dual space curve $\hat{x}$ supposed as the dual unit speed, then the moving dual frame $\{\overrightarrow{\hat{t}(s)}, \overrightarrow{\hat{n}(s)}, \overrightarrow{\hat{b}(s)}\}$ moves in accordance with (2). This motion consists of an instantaneous translation along $\hat{x}$ and an instantaneous rotation about $\hat{x}$. Then, the instantaneous rotation is determined by an angular velocity vector given by dual Darboux vector

$$
\overrightarrow{\hat{\partial}}=\hat{\tau} \hat{t}(s)+\hat{\varkappa} \overrightarrow{\hat{b}(s)}
$$

which satisfies

$$
\begin{aligned}
& \frac{d \overrightarrow{\hat{t}(s)}}{d \hat{s}}=\overrightarrow{\hat{\partial}} \times \overrightarrow{\hat{t}(s)}, \\
& \frac{d \hat{\hat{n}(s)}}{d \hat{s}}=\overrightarrow{\hat{\partial}} \times \overrightarrow{\hat{n}(s)}, \\
& \frac{d \hat{b}(s)}{d \hat{s}}=\overrightarrow{\hat{\partial}} \times \overrightarrow{\hat{b}(s)} .
\end{aligned}
$$

The direction of the dual Darboux vector is that of the instantaneous axis of rotation. Dual Darboux rotation of dual Frenet frame can be separated into two rotation motions (see [3]):

Dual tangent vector $\overrightarrow{\hat{t}(s)}$ rotates with a $\hat{\varkappa}$ angular speed round the dual binormal vector $\vec{b}(s)$, that is

$$
\frac{d \overrightarrow{\hat{t}(s)}}{d \hat{s}}=(\hat{\varkappa} \overrightarrow{\hat{b}(s)}) \times \overrightarrow{\hat{t}(s)}
$$

and the dual binormal vector $\overrightarrow{\hat{b}(s)}$ rotates with a $\hat{\tau}$ angular speed round the dual tangent vector $\overrightarrow{\hat{t}(s)}$, that is

$$
\frac{d \overrightarrow{\hat{b}(s)}}{d \hat{s}}=(\hat{\tau} \hat{t}(s)) \times \overrightarrow{\hat{b}(s)} .
$$

From the application of the second equation of (4), we know that the position dual vector of a rectifying dual space curve is always in the direction of the dual Darboux vector. Therefore, rectifying dual space curves can be interpreted kinematically as those dual space curves whose position dual vector field determines the axis of instantaneous rotation at each point of the dual space curve.

In the next theorem, for rectifying dual space curves we get a characterization in terms of the harmonic curvature $\frac{\hat{\tau}}{\hat{\imath}}$.

Theorem 2.2. Let $\hat{x}: I \rightarrow \mathbb{D}^{3}$ be a dual space curve with $\hat{\varkappa}=\varkappa+\varepsilon \varkappa^{*}$ such that $\varkappa>0$ and let $\hat{s}$ be the dual arc length of the dual space curve $\hat{x}$. Then $\hat{x}$ is congruent 
to a rectifying dual space curve if and only if the harmonic curvature $\frac{\hat{\tau}}{\hat{\kappa}}$ of the dual space curve $\hat{x}$ is a nonconstant linear function in dual arc length function $\hat{s}$, that is, $\hat{\hat{\tau}}=\hat{c}_{1} \hat{s}+\hat{c}_{2}$, where $\hat{c}_{1}=c_{1}+\varepsilon c_{1}^{*}, \hat{c}_{2}=c_{2}+\varepsilon c_{2}^{*} \in \mathbb{D}$ and $c_{1} \neq 0$.

Proof. Let $\hat{x}: I \rightarrow \mathbb{D}^{3}$ be a unit speed dual space curve with $\hat{\varkappa}=\varkappa+\varepsilon \varkappa^{*}$, in which $\varkappa>0$. If $\hat{x}$ is a rectifying dual space curve, then by the proof of Theorem 2.1 and by relations (4) and (5), we have

$$
\frac{\hat{\tau}}{\hat{\varkappa}}=\frac{\hat{s}+\hat{k}}{\hat{a}},
$$

where $\hat{a}$ is a dual constant. Hence, the harmonic curvature $\frac{\hat{\tau}}{\hat{\imath}}$ of the dual space curve $\hat{x}$ is a nonconstant linear function of the dual arc length function $\hat{s}$.

Conversely, let us suppose that the harmonic curvature $\frac{\hat{\tau}}{\hat{\kappa}}$ is a nonconstant linear function such that $\frac{\hat{\tau}}{\hat{\varkappa}}=\hat{c}_{1} \hat{s}+\hat{c}_{2}$, where $\hat{c}_{1}, \hat{c}_{2} \in \mathbb{D}$ and $\hat{c}_{1}$ is nowhere pure dual. If we take $\hat{a}=\frac{1}{\hat{c}_{1}}$ and $\hat{k}=\hat{a} \hat{c}_{2}$, then we get $\frac{\hat{\tau}}{\hat{\varkappa}}=\frac{\hat{s}+\hat{k}}{\hat{a}}$. Thus, by using Frenet equations (2), we find that

$$
\frac{d}{d \hat{s}}[\overrightarrow{\hat{x}(s)}-(\hat{s}+\hat{k}) \overrightarrow{\hat{t}(s)}-\hat{a} \hat{b}(s)]=0,
$$

which means that the dual space curve $\hat{x}$ is congruent to a rectifying dual space curve.

\section{Classification of rectifying dual space curves}

Now we give a theorem determining the parameterization of a unit speed rectifying curve in $\mathbb{D}^{3}$.

Theorem 3.1. Let $\hat{x}: I \rightarrow \mathbb{D}^{3}$ be a dual space curve with $\hat{\varkappa}=\varkappa+\varepsilon \varkappa^{*}$ such that $\varkappa>0$. Then, the dual space curve $\hat{x}$ is a rectifying dual space curve if and only if, up to parameterization, $\hat{x}$ is given by

$$
\hat{x}(t)=(\hat{a} \sec \hat{t}) \hat{y}(t)
$$

where $\hat{a}=a+\varepsilon a^{*}(a>0)$ is a dual number and $\hat{y}=\hat{y}(t)$ a dual unit speed curve in $\mathbb{S}^{2}$.

Proof. Let us suppose that the dual curve $\hat{x}$ is a rectifying dual space curve with $\hat{\varkappa}=\varkappa+\varepsilon \varkappa^{*}$ such that $\varkappa>0$ and that $\hat{x}=\hat{x}(s)$ is a dual unit speed curve. From Theorem 2.1 we know that the dual distance function $\hat{\rho}=\|\overrightarrow{\hat{x}(s)}\|$ satisfies $\hat{\rho}^{2}=$ $\hat{s}^{2}+\hat{c}_{1} \hat{s}+\hat{c}_{2}$ for some dual constants $\hat{c}_{1}$ and $\hat{c}_{2}$. After making a suitable translation in $\hat{s}$, we obtain $\hat{\rho}^{2}=\hat{s}^{2}+\hat{c}$ for some dual constant $\hat{c}=c+\varepsilon c^{*}$, where $c>0$. Therefore, we may choose $\hat{c}=\hat{a}^{2}$. Next, we define a dual space curve in the dual unit sphere $\mathbb{S}^{2}$ by $\hat{y}=\frac{\hat{x}}{\hat{\rho}}$. Hence, we have

$$
\hat{x}(s)=\left(\hat{s}^{2}+\hat{a}^{2}\right)^{\frac{1}{2}} \hat{y}(s) .
$$


If we take the derivative of equation (8) with respect to $\hat{s}$, we get

$$
\frac{d \hat{x}(s)}{d \hat{s}}=\frac{\hat{s}}{\sqrt{\hat{s}^{2}+\hat{a}^{2}}} \hat{y}(s)+\sqrt{\hat{s}^{2}+\hat{a}^{2}} \frac{d \hat{y}(s)}{d \hat{s}} .
$$

On the other hand, since $\langle\hat{y}(s), \hat{y}(s)\rangle=1,\left\langle\hat{y}(s), \frac{d \hat{y}(s)}{d \hat{s}}\right\rangle=0$. Thus, by taking into consideration that $\hat{x}=\hat{x}(s)$ is a dual unit speed curve in (9), we find $\left\|\frac{d \hat{y}(s)}{d \hat{s}}\right\|=\frac{\hat{a}}{\hat{s}^{2}+\hat{a}^{2}}$. Then,

$$
\hat{t}=\int_{o}^{\hat{s}} \frac{\hat{a}}{\hat{u}^{2}+\hat{a}^{2}} d \hat{u}=\arctan \left(\frac{\hat{s}}{\hat{a}}\right) .
$$

So, $\hat{s}=\hat{a} \tan \hat{t}$. By using this in (8), we have (7).

Conversely, if $\hat{x}: I \rightarrow \mathbb{D}^{3}$ is a dual space curve defined by

$$
\hat{x}(t)=(\hat{a} \sec \hat{t}) \hat{y}(t)
$$

for a dual number $\hat{a}=a+\varepsilon a^{*}(a>0)$ and a dual unit speed curve $\hat{y}=\hat{y}(t)$ in $\mathbb{S}^{2}$, then we get

$$
\frac{d \hat{x}(t)}{d \hat{t}}=(\hat{a} \sec \hat{t})\left\{(\tan \hat{t}) \hat{y}(t)+\frac{d \hat{y}(t)}{d \hat{t}}\right\}
$$

Since $\hat{y}(t)$ and $\frac{d \hat{y}(t)}{d \hat{t}}$ are orthonormal vector fields, from (11) we find

$$
\left\|\frac{d \hat{x}(t)}{d \hat{t}}\right\|=\hat{a} \sec ^{2} \hat{t}
$$

From equations (10), (11), and (12), we get

$$
\left\langle\hat{x}^{N}, \hat{x}^{N}\right\rangle=\hat{\rho}^{2}(t)-\frac{\left\langle\hat{x}(t), \frac{d \hat{x}(t)}{d \hat{t}}\right\rangle^{2}}{\left\|\frac{d \hat{x}(t)}{d \hat{t}}\right\|^{2}}=\hat{a}^{2},
$$

which shows that the normal component $\hat{x}^{N}$ of the position dual vector has constant length. Hence, from Theorem 2.1 it is seen that $\hat{x}$ is a rectifying dual space curve.

We know that oriented lines in a Euclidean three-space $\mathbb{R}^{3}$ may be represented by unit vectors with three components over the ring of dual numbers. A differentiable curve on the dual unit sphere depending on a real parameter $v$ corresponds to a ruled surface in $\mathbb{R}^{3}$. This correspondence is one to one and allows the geometry of ruled surfaces to be represented by the geometry of dual spherical curves on a unit dual sphere. Dual spherical geometry, expressed with the aid of unit dual vectors, is closely analogous to real spherical geometry, expressed with the aid of unit real vectors. Therefore, the properties of elementary real spherical geometry can be carried over by analogy into the geometry of lines $\mathbb{R}^{3}$ (see $[4,5]$ ).

In the light of all mentioned above, the following corollary gives a link between our work and the classical surface theory. 
Corollary 3.2. Let $\hat{x}: I \rightarrow \mathbb{D}^{3}$ be a rectifying dual space curve. Then,

$$
\frac{1}{\hat{a}} \cos \hat{t} \hat{x}(t)
$$

corresponds to a ruled surface in $\mathbb{R}^{3}$.

\section{References}

[1] B.-Y. Chen, When does the position vector of a space curve always lie in its rectifying plane?, Amer. Math. Monthly 110 (2003), no. 2, 147-152.

[2] B.-Y. Chen and F. Dillen, Rectifying curves as centrodes and extremal curves, Bull. Inst. Math. Acad. Sinica 33 (2005), no. 2, 77-90.

[3] A. C. Çöken and A. Görgülü, On the dual Darboux rotation axis of the dual space curve, Demonstratio Math. 35 (2002), no. 2, 385-390.

[4] H. W. Guggenheimer, Differential geometry, McGraw-Hill Book Co., New York, 1963.

[5] H. H. Hacısalihoğlu, On the pitch of a closed ruled surface, Mech. Mach. Theory 7 (1972), no. 3, 291-305.

[6] Ö. Köse, Ş. Nizamoğlu, and M. Sezer, An explicit characterization of dual spherical curves, Doğa Mat. 12 (1988), no. 3, 105-113.

[7] G. R. Veldkamp, On the use of dual numbers, vectors and matrices in instantaneous, spatial kinematics, Mech. Mach. Theory 11 (1976), no. 2, 141-156. 